\title{
CONFINED PARTIAL FILAMENT ERUPTION AND ITS REFORMATION WITHIN A STABLE MAGNETIC FLUX ROPE
}

\author{
Navin Chandra Joshi ${ }^{1,6}$, Abhishek K. SRivastava ${ }^{2}$, Boris Filippov ${ }^{3}$, Pradeep Kayshap ${ }^{1}$, \\ Wahab Uddin ${ }^{1}$, Ramesh Chandra $^{4}$, Debi Prasad Choudhary ${ }^{5}$, and B. N. Dwivedi ${ }^{2}$ \\ ${ }^{1}$ Aryabhatta Research Institute of Observational Sciences (ARIES), Manora Peak, Nainital 263 002, Uttarakhand, India; navin@aries.res.in, njoshi98@gmail.com \\ ${ }^{2}$ Department of Physics, Indian Institute of Technology (Banaras Hindu University), Varanasi 221005, India \\ ${ }^{3}$ Pushkov Institute of Terrestrial Magnetism, Ionosphere and Radio Wave Propagation, Russian Academy of Sciences, Troitsk, Moscow, Russia \\ ${ }^{4}$ Department of Physics, D.S.B. Campus, Kumaun University, Nainital 263 002, Uttarakhand, India \\ ${ }^{5}$ California State University Northridge, 18111 Nordhoff Street, Northridge, CA 91330, USA \\ Received 2013 August 7; accepted 2014 March 11; published 2014 April 29
}

\begin{abstract}
We present observations of a confined partial eruption of a filament on 2012 August 4, which restores its initial shape within $\approx 2 \mathrm{hr}$ after eruption. From the Global Oscillation Network Group $\mathrm{H} \alpha$ observations, we find that the filament plasma turns into dynamic motion at around 11:20 UT from the middle part of the filament toward the northwest direction with an average speed of $\approx 105 \mathrm{~km} \mathrm{~s}^{-1}$. A little brightening underneath the filament possibly shows the signature of low-altitude reconnection below the filament eruptive part. In Solar Dynamics Observatory/Atmospheric Imaging Assembly $171 \AA$ images, we observe an activation of right-handed helically twisted magnetic flux rope that contains the filament material and confines it during its dynamical motion. The motion of cool filament plasma stops after traveling a distance of $\approx 215 \mathrm{Mm}$ toward the northwest from the point of eruption. The plasma moves partly toward the right foot point of the flux rope, while most of the plasma returns after 12:20 UT toward the left foot point with an average speed of $\approx 60 \mathrm{~km} \mathrm{~s}^{-1}$ to reform the filament within the same stable magnetic structure. On the basis of the filament internal fine structure and its position relative to the photospheric magnetic fields, we find filament chirality to be sinistral, while the activated enveloping flux rope shows a clear right-handed twist. Thus, this dynamic event is an apparent example of one-to-one correspondence between the filament chirality (sinistral) and the enveloping flux rope helicity (positive). From the coronal magnetic field decay index, $n$, calculation near the flux rope axis, it is evident that the whole filament axis lies within the domain of stability (i.e., $n<1$ ), which provides the filament stability despite strong disturbances at its eastern foot point.
\end{abstract}

Key words: Sun: corona - Sun: filaments, prominences - Sun: magnetic fields

Online-only material: animations, color figures

\section{INTRODUCTION}

Filaments/prominences are relatively cooler and denser plasma structures imbedded in the hot ambient corona. These magnetic structures show up as dark curtains (filaments) on the bright solar disk and as bright features on the limb against the dark background (i.e., prominences). Filaments are associated with different chiralities depending on the direction of their axial magnetic fields relative to the distribution of photospheric magnetic fields. If the axial field direction is rightward/leftward when viewed from the positive polarity side, it is defined as the dextral/sinistral (Martin 1998a). In addition, there are other morphological features that also characterize the axial field direction and the kind of chirality, such as filament end points, filament barbs, and coronal arcades (see Martin 1998a). Filament chirality and its association with magnetic helicity have been extensively studied (see Martin 1998a; Rust 1999; Chae 2000; Mackay et al. 2000; Pevtsov et al. 2003; Chandra et al. 2010). The evidence of mixed helicity in eruptive filaments has been reported by Muglach et al. (2009).

Filaments generally exist in the equilibrium state because of the balance between the upward magnetic pressure and the downward magnetic tension forces and gravity (Chen 2011, and references therein). Various theories have been proposed for the formation of filaments and their eruptions (Martin 1998a, 1998b; Rust 2001; Mackay et al. 2010; Labrosse et al. 2010;

\footnotetext{
6 Present address: School of Space Research, Kyung Hee University, Yongin, Gyeonggi-Do, 446-701, Korea.
}

Schmieder et al. 2010, and references therein). Some theories, based on observational studies, suggest the reconfiguration of a pre-existing coronal magnetic field along the polarity inversion line (PIL) which plays a key role in the formation of a filament through surface motion, flux convergence, flux cancellation, etc. (Gaizauskas et al. 1997; Martin 1998a; Gaizauskas et al. 2001, and references therein). Shearing of the magnetic field due to photospheric motions distorts/disturbs the stability of a filament, resulting in a filament eruption. Some authors have discussed a new paradigm, suggesting that a filament is a consequence of the emergence of a toroidal magnetic flux rope generated by subsurface motions inside the Sun (Lites \& Low 1997; Lites 2005; Rust 2001). Sufficient accumulation of twist makes the filament unstable and leads to its eruption.

Due to sufficient twist accumulation and force imbalance, filaments show different kinds of eruptions, e.g., failed, partially failed, or full eruptions (Török \& Kliem 2005; Tripathi et al. 2009; Joshi et al. 2013a). Magnetic flux emergence in an active region is responsible for the flux imbalance and storage of free magnetic energy, which also causes the solar eruptions and flares (Sterling et al. 2007; Chandra et al. 2009; Choudhary et al. 2013). Some filament eruptions are followed by two ribbon flares and coronal mass ejections (CMEs), while others are not (Choudhary \& Moore 2003; Chandra et al. 2011). During a full eruption, all of the magnetic field and plasma eject out into the interplanetary space in the form of a CME (Sterling et al. 2012; Chandra et al. 2010; Joshi et al. 2013b). In the case of failed eruption, however, material and the magnetic field remain confined in the corona (Ji et al. 2003; Török \& Kliem 2005; Liu 
et al. 2009; Kumar et al. 2011; Joshi et al. 2013a; Kuridze et al. 2013). Partial filament eruption is characterized as an eruption during which some of the material erupts while some of it stays in equilibrium or falls back onto the Sun (Tripathi et al. 2009; Shen et al. 2012). Failed filament eruptions have been observed and modeled by various authors (Filippov \& Koutchmy 2002; Török \& Kliem 2005; Liu et al. 2009; Kumar et al. 2011; Joshi et al. 2013a). The overlying magnetic configuration is known to be responsible for failed or partially failed eruptions (Liu et al. 2009; Kumar et al. 2011; Filippov 2013). Liu et al. (2009) reported a failed eruption and interpreted it, invoking the presence of the overlying asymmetric magnetic field. Kumar et al. (2011) studied a failed flux rope eruption and suggested that the overlying remnant filament and its magnetic field were responsible for this failed eruption. Filippov (2013) studied a filament eruption on 2010 October 21 and calculated the potential magnetic field decay index near the filament. He has suggested that, after having reached a height in the corona where the decay index was greater than one, the filament was ready to erupt. Recently, Joshi et al. (2013a) reported the collapse of a CME core caused by an asymmetric filament eruption and made an effort to interpret it using a self-consistent model of a magnetic flux rope. Apart from these observations, several simulations have been carried out to understand the stability of filaments and their eruptions (Schmieder et al. 1985; Filippov et al. 2001; Török \& Kliem 2005; DeVore \& Antiochos 2008; Tripathi et al. 2009; Fan 2010; Schmieder et al. 2013). In some of these flux rope equilibrium models, it has been concluded that the rate of decrease of surrounding magnetic field with height plays a crucial role in the equilibrium and stability of a flux rope (van Tend \& Kuperus 1978; Molodenskii \& Filippov 1987; Filippov \& Den 2001; Kliem \& Török 2006; Démoulin \& Aulanier 2010).

A reformation of filament is yet another important feature that is often observed after filament eruptions. These reformations occur on diverse timescales ranging from a few minutes to days (Gilbert et al. 2007; DeVore \& Antiochos 2008; Tripathi et al. 2009; Koleva et al. 2012). Koleva et al. (2012) studied the evolution and eruption of a helical loop-like prominence along with its partial reformation two days later in the same region during 2010 March 31 to 2010 April 2. Gilbert et al. (2007) extensively discussed the dynamics of kinking processes in filaments and their implications for failed, partial, and full eruptions, along with the reformations. DeVore \& Antiochos (2008) simulated the confined homologous filament eruptions and their reformation in a quadrupolar magnetic configuration. Tripathi et al. (2009) studied six cases of partial eruptions and found that the signature of filament reformation is evidence of surviving filament material. Recently, Li \& Zhang (2013) reported an observation taken on 2012 August 4 of fine structures and magnetic properties of the filament-associated flux rope. However, they did not consider the failed nature of its eruption and its reformation.

In this paper, we present observational evidence of the dynamic stability of the flux rope with the confined partial filament eruption (disappearance) within it on 2012 August 4 and the following reformation of the filament in the same location. We study the role of the surrounding magnetic field for the stability of the flux rope and possibility of reformation of the disappeared part of the filament. Observational data sets are presented in Section 2. The corresponding observational results are discussed in Section 3. Coronal magnetic field calculations and their use for interpretation of the flux rope stability during the confined eruption and reformation are discussed in Section 4. Results and discussion are given in the last section (i.e., Section 5).

\section{OBSERVATIONAL DATA SET}

We use data from the Solar Dynamics Observatory (SDO)/ Atmospheric Imaging Assembly (AIA), SDO/Helioseismic and Magnetic Imager (HMI), the Solar Terrestrial Relations Observatory (STEREO)/SECCHI/EUVI, Global Oscillation Network Group (GONG), and Big Bear Solar Observatory (BBSO) for this study. The brief details of these instruments and their data are as follows.

(i) BBSO and NSO-GONG H $\alpha$ Data. We used $\mathrm{H} \alpha$ data from $\mathrm{BBSO}^{7}$ to investigate the chirality of the filament on the solar disk based on fine structure on the main filament body and its barbs. $\mathrm{H} \alpha$ observations for the study of temporal evolution have been taken from the GONG network archive. ${ }^{8}$ GONG collected $\mathrm{H} \alpha$ images at seven sites over the globe in the $6563 \AA$ wavelength with a spatial resolution of $1^{\prime \prime}$ and a cadence of around 1 minute (Harvey et al. 2011). The $\mathrm{H} \alpha$ observations are used to investigate the kinematics of cool material within the filament channel.

(ii) SDO AIA and HMI Data. AIA is an instrument on board the $S D O$. It observes the full solar disk in three ultraviolet (UV) continuum wavelengths and seven extreme ultraviolet (EUV) narrowband wavelengths (Lemen et al. 2012). $S D O /$ AIA has a pixel size of 0.6 and a minimum cadence of $12 \mathrm{~s}$. Therefore, it provides observations of multitemperature, high-resolution, and high-temporal plasma dynamics across the whole Sun. In our study, we use $171 \AA$ images to study the activation of right-handed helically twisted flux rope that warp the cool filament material. HMI (Schou et al. 2012) is another instrument on board SDO. It provides full disk magnetograms of the Sun with $1^{\prime \prime}$ spatial resolution and with a minimum cadence of $45 \mathrm{~s}$. We used SDO/HMI magnetogram data to find the magnetic polarity distribution near the filament channel as well as to calculate the decay index at various heights in the corona.

(iii) STEREO-B/SECCHI Data. SECCHI/EUVI is an instrument on board STEREO (Wuelser et al. 2004; Howard et al. 2008). It observes the full solar disk in four wavelengths, viz. $304 \AA, 195 \AA, 284 \AA$, and $171 \AA$, with a spatial resolution of 1".6. SECCHI/EUVI $195 \AA$ data have been used for the investigation of the activation of twisted flux rope from different angles as STEREO-B provides the limb view of the particular eruptive region.

\section{OBSERVATIONS OF THE DYNAMICS OF THE FILAMENT AND FLUX ROPE}

This event was observed in several spectral lines with different instruments, which provides a unique opportunity to study the filament dynamics in multitemperature plasma and in different projections. SDO/AIA and GONG data provide ondisk observations of the event, while STEREO-B/SECCHI provides observations of the limb. Figure 1 (top panel) shows the filament in the $\mathrm{H} \alpha$ line observed from GONG instruments at $\approx 11: 00$ UT on 2012 August 4. The top left panel (Figure 1(a)) of this figure shows the GONG full disk image on 2012 August 4 , with a box showing the location of the filament. The zoomed image of the filament in $\mathrm{H} \alpha$ is shown in the right panel

\footnotetext{
7 http://www.bbso.njit.edu/

8 http://halpha.nso.edu/archive.html
} 

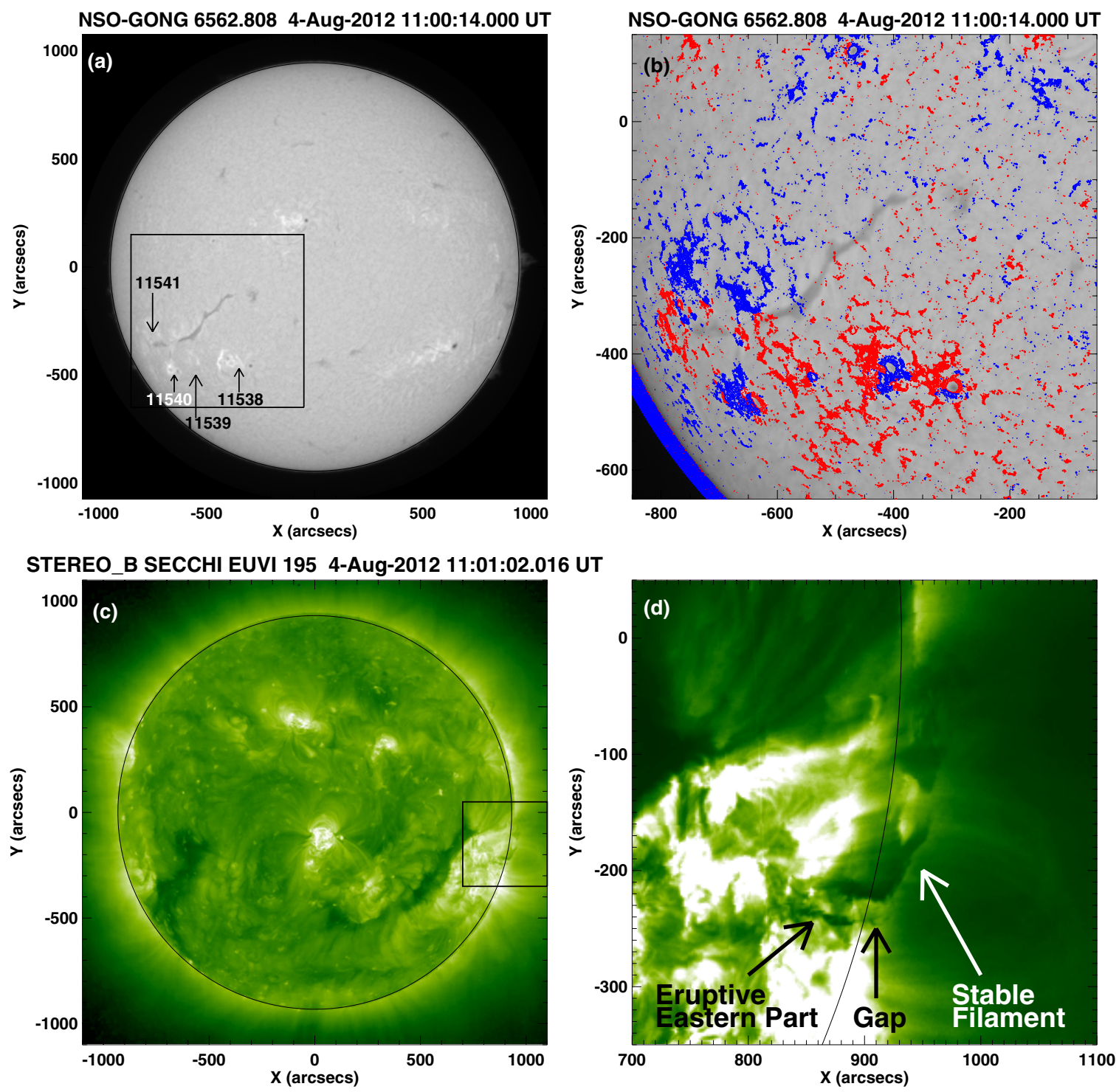

Figure 1. (a) Full disk $\mathrm{H} \alpha$ image from the Global Oscillation Network Group (GONG) in which the box shows the location of the filament. (b) Selected area of the GONG-H $\alpha$ network showing the filament on the disk. In the panels (b), the contours are the HMI magnetogram contours at $\pm 50, \pm 100, \pm 200, \pm 300$, and $\pm 400 \mathrm{G}$. The red (blue) contour shows positive (negative) polarity regions. (c) Full disk STEREO/EUVI $195 \AA$ A images in which the box shows the location of the filament over the limb. (d) Zoomed view of the filament corresponding to the box in panel 1(c).

(A color version of this figure is available in the online journal.)

(Figure 1(b)) with the overplotted SDO/HMI magnetogram contours. It is clear that the filament lies along the PIL on the solar disk. The left foot point is anchored in the stronger photospheric field compared to the right foot point of the filament, suggesting that the flux may not be uniform along the filament channel. The full disk STEREO-B EUVI $195 \AA$ image, which shows the limb view of the filament, is represented in Figure 1(c). The zoomed view of the filament within the black box is shown in Figure 1(d). The filament lies between the active regions NOAA AR 11541, AR 11540, and AR 11538 (see Figure 1(a)).

Recently, Li \& Zhang (2013) studied fine structures in the flux rope, which became visible after the filling of flux tubes by heated filament material from the eastern part of the filament channel. They also estimated various parameters of this archshaped flux rope with a total length of around $\approx 546 \mathrm{Mm}$ (from the left east foot point to the right west foot point). This flux rope contains $\approx 102 \pm 15$ fine structures with a width ranging from $1^{\prime \prime} .1$ to $1^{\prime \prime} .7$. They also identified 22 western foot points of the fine structures that lie within positive polarity with the net magnetic flux ranging from $1.1 \times 10^{19} M_{X}$ to $8.1 \times 10^{19} M_{X}$.

Figure 2 shows the high-resolution image of the filament cold plasma observed in the $\mathrm{H} \alpha$ line by BBSO. This high-resolution image is useful for finding the chirality of the filament. The filament has three barbs, which are marked by white arrows in Figure 2. In most cases, the barbs of a dextral/sinistral filament are observed to be right/left bearing (Martin 1998a). The direction of thin threads within the main body of the filament often shows the direction of the magnetic field within the filament, which is related to chirality. From Figure 2, it is clear that the filament barbs are left bearing, which corresponds to sinistral chirality. Thin threads within the main body of the filament deviate counterclockwise from the filament axis, which also corresponds to sinistral chirality.

The event started around 11:00 UT with the eruption of a short eastern section of the huge filament (see Figure 3, upper panel). A small $\mathrm{C}$ class flare was associated with this eruption. The eruption of the small eastern filament was observed by 


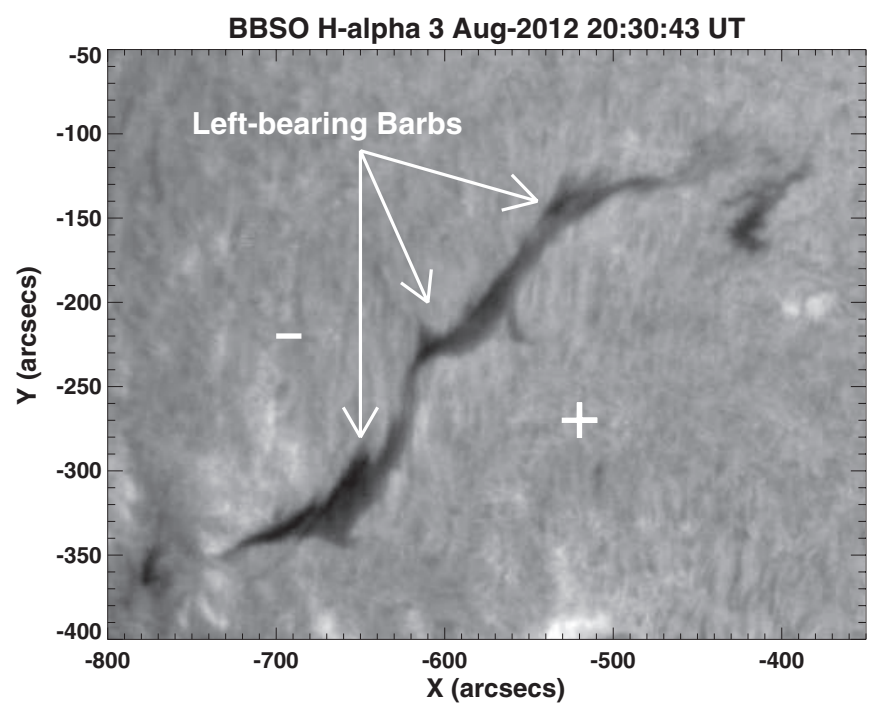

Figure 2. $\mathrm{H} \alpha$ image of the filament on 2012 August 3 at $\approx 20: 30: 43 \mathrm{UT}$ as observed from Big Bear Solar Observatory (BBSO). The + and - signs indicate the positive and negative polarity regions. White arrows indicate the left-bearing barbs of the filament.
STEREO-B/SECCHI and SDO/AIA. It produced a CME which was observed by both the Large Angle and Spectrometric Coronagraph C2, C3 on board the Solar and Heliospheric Observatory (Brueckner et al. 1995) and the coronagraph COR 2 (Howard et al. 2008) on board STEREO (not shown here). The eruption also caused the simultaneous activation of a large filament to the west of the eruption place and a large-scale flux rope containing the filament. Given that the CME perturbs the overlying large-scale coronal field, the equilibrium of the western section of stable flux rope may have been perturbed as well, so that the western section began to move to a new equilibrium at a different height.

However, this eastern eruptive filament was separated by a gap from the main body of the filament and might be considered as a small independent filament (see Figure 3, upper panel). Both parts were located above the same photospheric PIL. At a greater height, the PIL changes its connectivity and forms two independent contours (PIL reconnection, bottom panel of Figure 3). This peculiarity of the potential magnetic field is possibly the reason for the existence of the gap between the eastern section and the main body of the filament on all the days of observations from 2012 August 3 to 6. Here, we have used the HMI magnetogram taken on 2012 August 5 for more reliable data at the place of the gap, because on 2012 August 4,
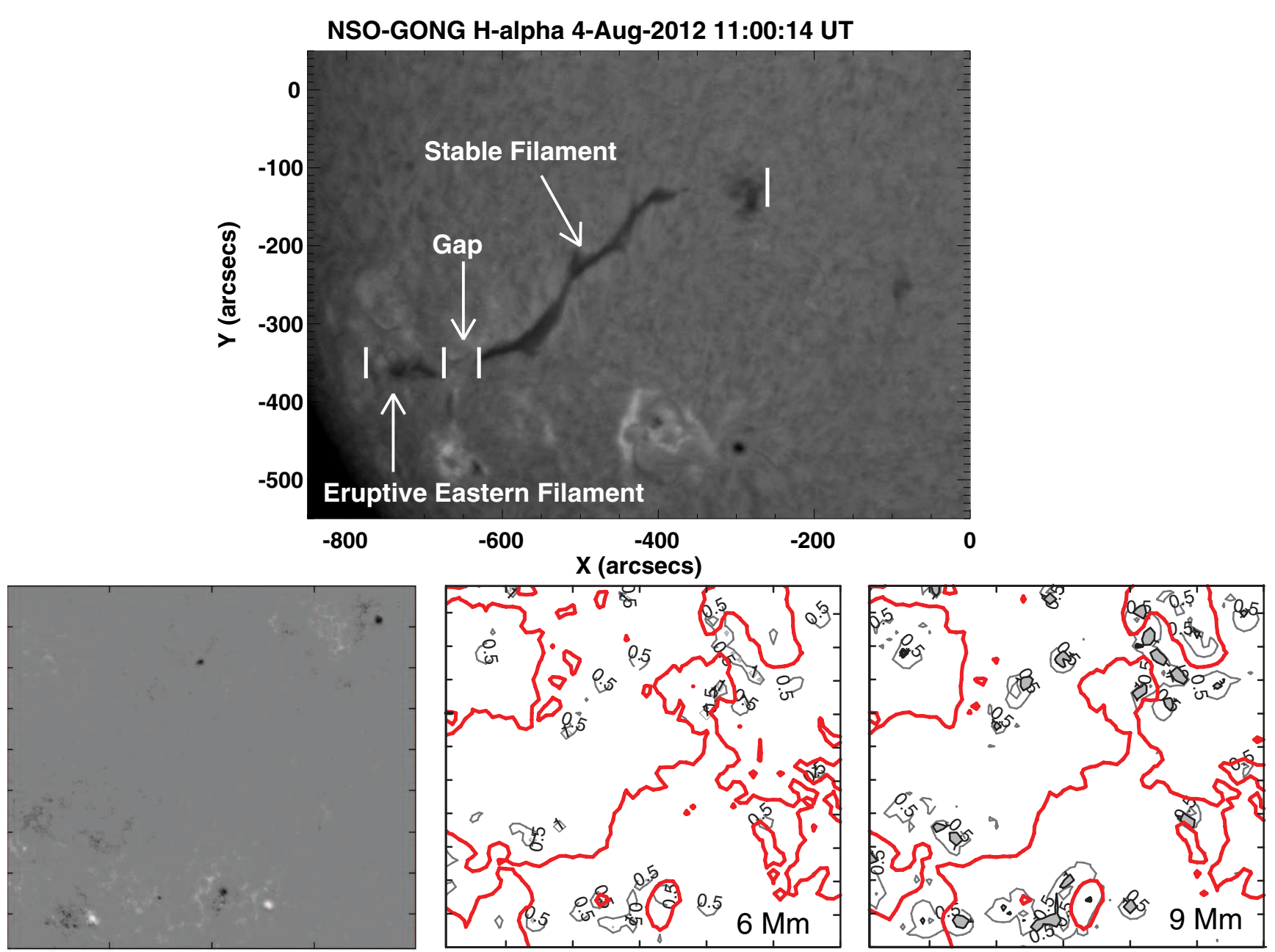

Figure 3. Upper panel: GONG $\mathrm{H} \alpha$ image at 11:00:14 UT on 2012 August 4 showing the eruptive and stable filament parts and the gap between them. Bottom panel: a fragment of the HMI magnetogram on 2012 August 5 (left) and PILs of the potential field at the height of $6 \mathrm{Mm}$ (middle) and 9 Mm (right). The PIL associated with the filament changes connectivity at the left bottom part of the frame where there is a gap in the filament.

(A color version of this figure is available in the online journal.) 


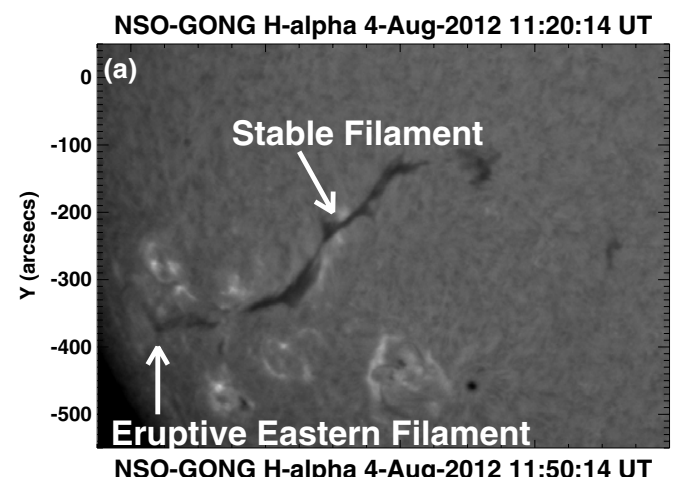

NSO-GONG H-alpha 4-Aug-2012 11:30:14 UT
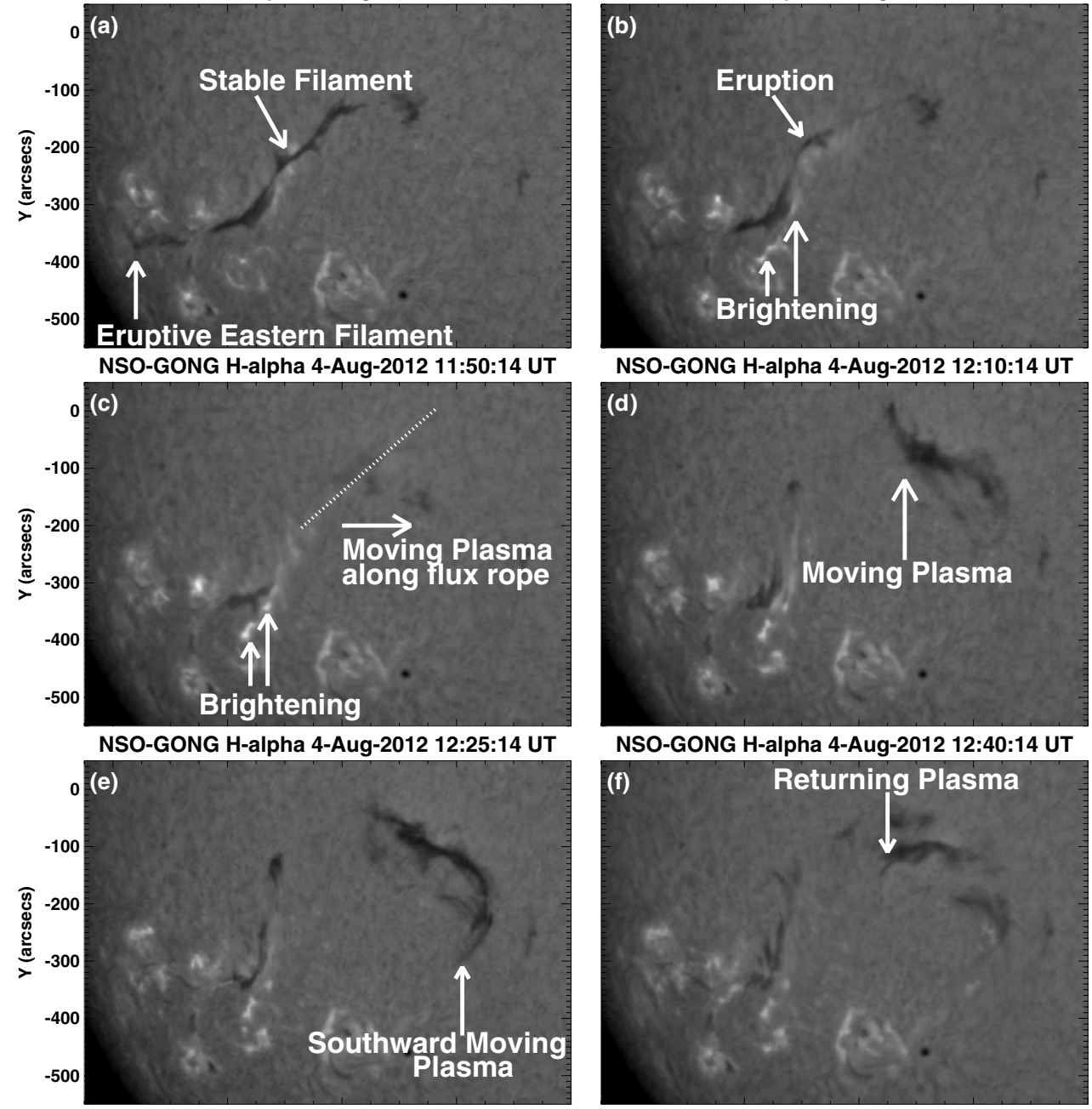

NSO-GONG H-alpha 4-Aug-2012 13:30:14 UT
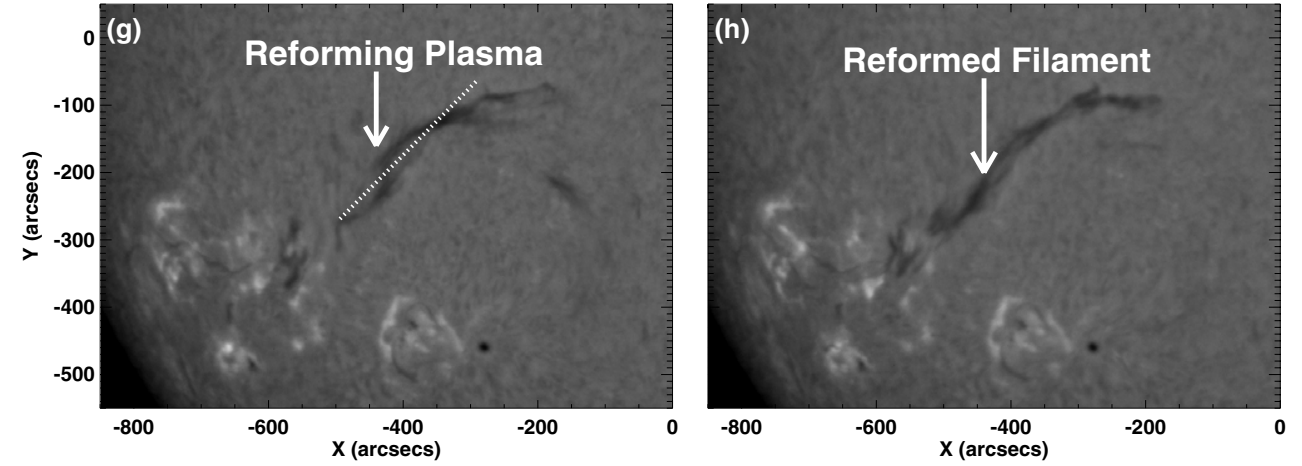

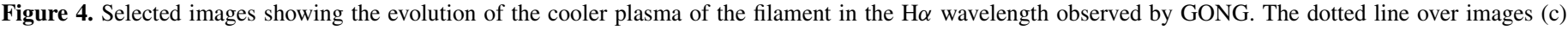

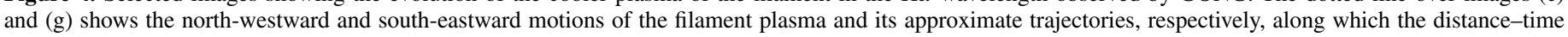
measurements have been performed.

(An animation of this figure is available in the online journal.)

this area was rather close to the limb. However, the photospheric magnetic field did not change significantly from 2012 August 4 to 5, so the results of the potential magnetic field calculations are nearly the same as for 2012 August 4 (see Figure 10 later). The details of the magnetic field and PIL calculations are presented in Section 4. We are interested in the study of the western part of the filament on 2012 August 4, including its stability and reformation. After the eastern filament eruption and the flare, hot plasma filled the thin threads of the filament flux rope and made them observable. They were practically invisible before the activation and constituted a coronal cavity around the filament. The details of the filament evolution and plasma dynamics, as well as their possible physical interpretation, are described in the upcoming sections/subsections.

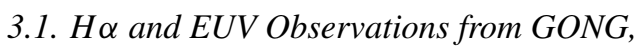 SDO/AIA, and STEREO/EUVI}

Figures 4 and 5 represent the sequence of selected GONG $\mathrm{H} \alpha$ and SDO/AIA 171 Å images, respectively. Dense plasma within 


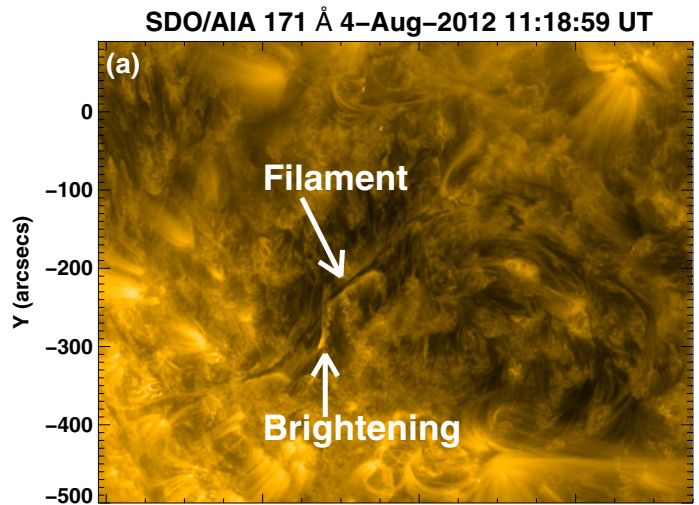

SDO/AIA 171 Å 4-Aug-2012 11:49:59 UT

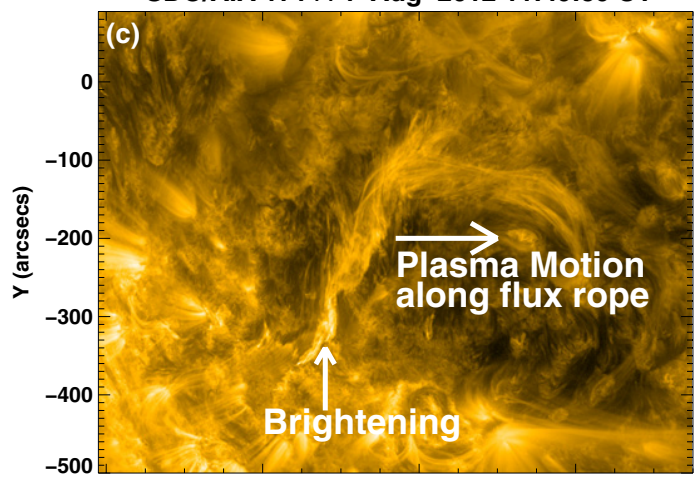

SDO/AIA $171 \AA$ A-Aug-2012 12:09:59 UT

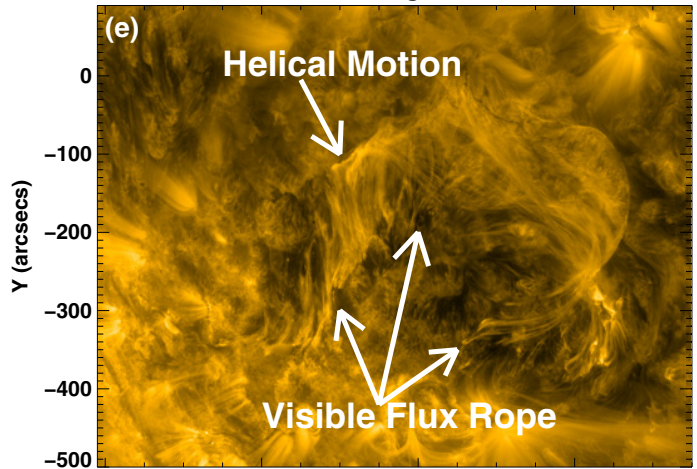

SDO/AIA $171 \AA \AA$ 4-Aug-2012 12:39:59 UT

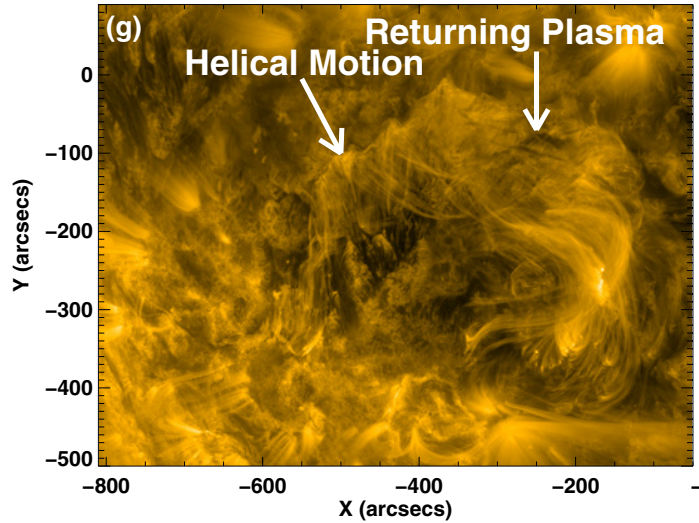

SDO/AIA 171 Å 4-Aug-2012 11:29:59 UT

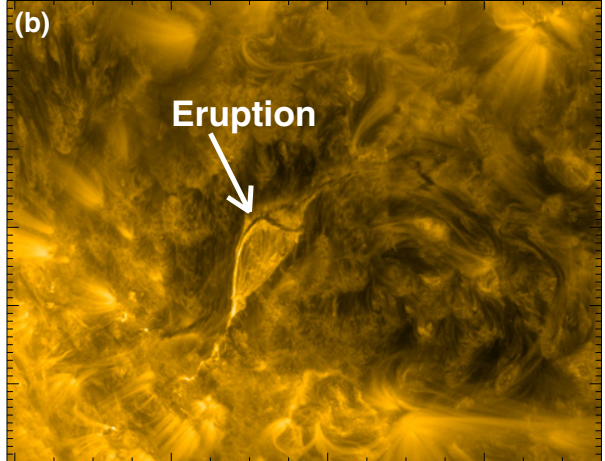

SDO/AIA 171 Å 4-Aug-2012 11:59:59 UT

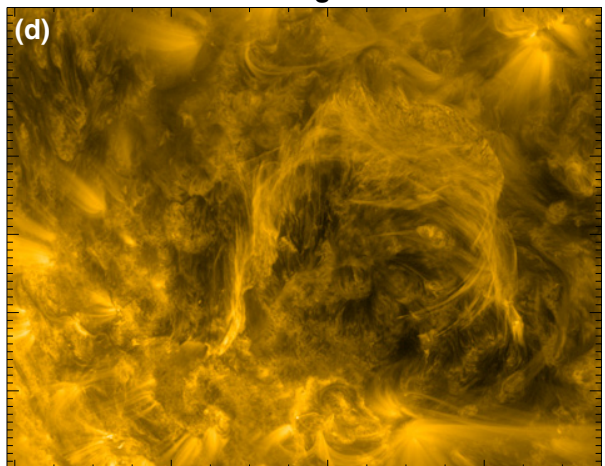

SDO/AIA $171 \AA \AA$ 4-Aug-2012 12:20:59 UT

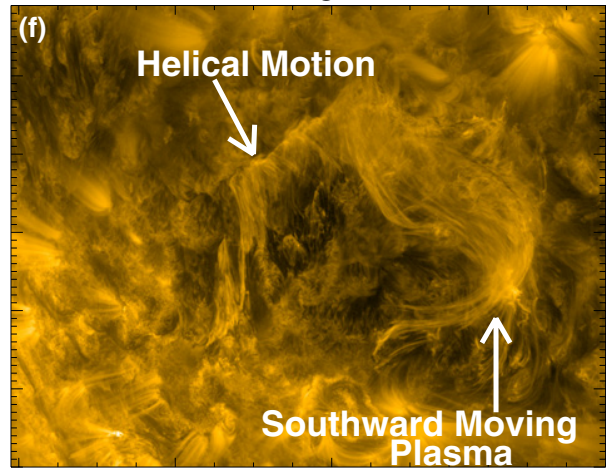

SDO/AIA $171 \AA ̊$ 4-Aug-2012 12:59:59 UT

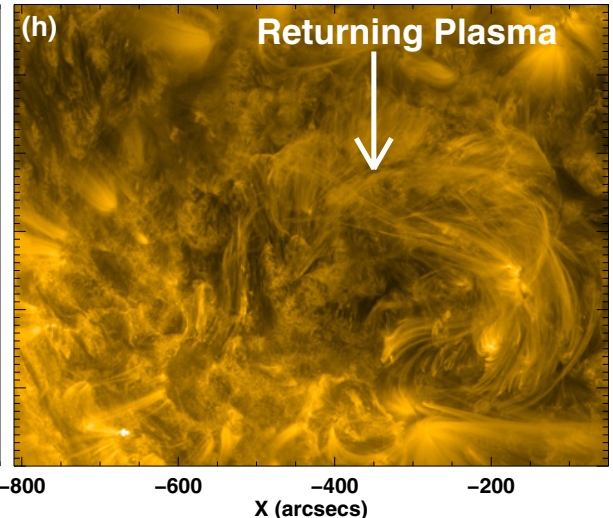

Figure 5. Selected SDO/AIA 171 A images showing the activation of the right-handed helically twisted flux rope containing the filament. (An animation and a color version of this figure are available in the online journal.)

the filament channel is most visible in the $\mathrm{H} \alpha$ line carrying the signature of the cooler chromosphere. SDO/AIA images reveal complicated plasma motion within the flux rope that represents the plasma formed at a temperature of $\sim 0.6 \mathrm{MK}$. Both
SDO/AIA $171 \AA$ and STEREO/EUVI $195 \AA$ A images provide information about the coronal plasma. A long forward J-shaped filament is visible on the southeast part of the solar disk (see Figures 4(a) and 5(a)) with sinistral chirality. The eastern 

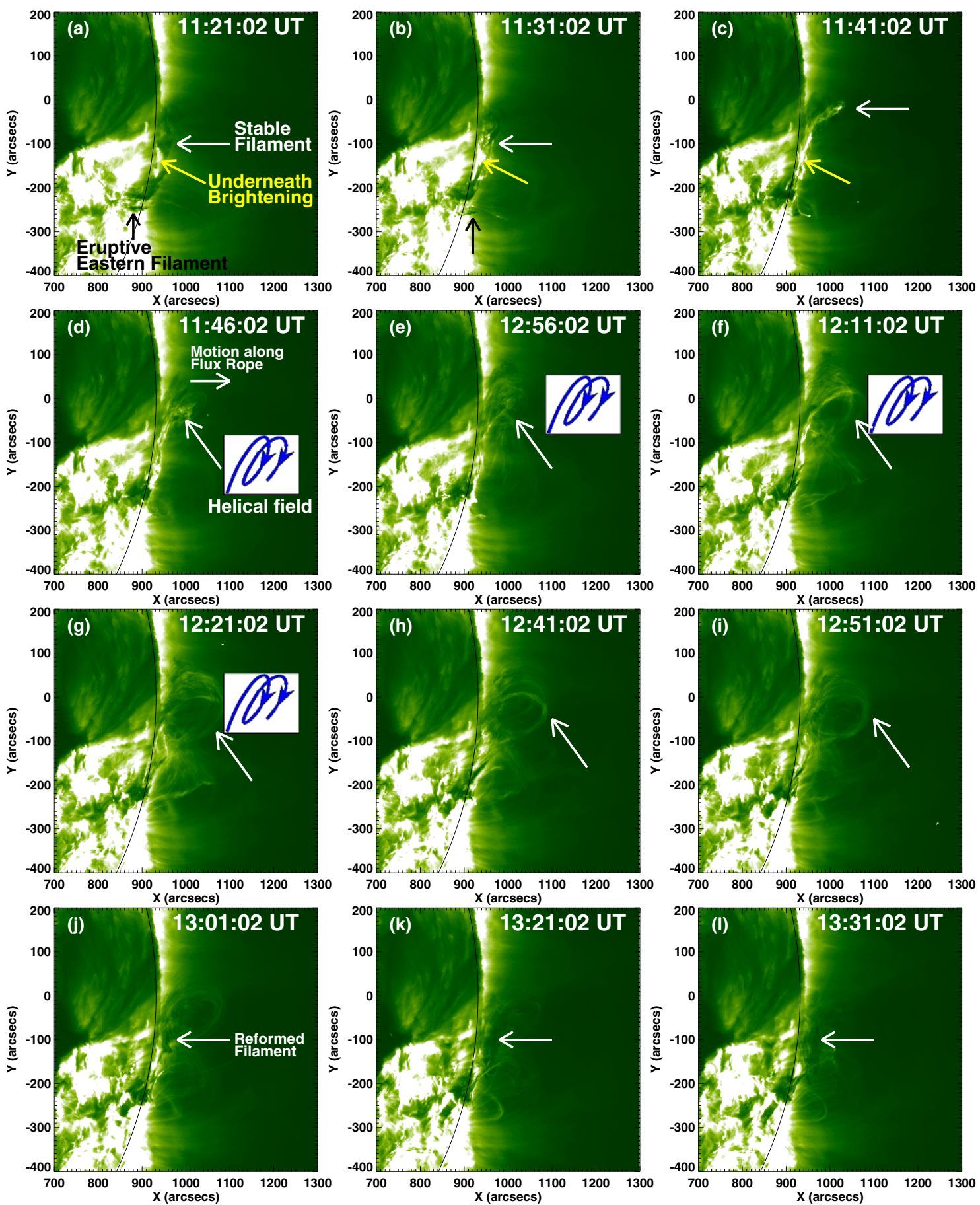

Figure 6. Evolution of the filament and flux rope activation in the STEREO/SECCHI/EUVI 195 A wavelength.

(An animation and a color version of this figure are available in the online journal.)

leg of this filament lies between the active regions NOAA 11541 and 11540, while the western part lies in a quiet region (see Figure 1(a)). From $\mathrm{H} \alpha$ observations, we estimated the approximate length of the visible filament to be $\approx 320 \mathrm{Mm}$ (see Figure 3(a) upper panel). Figure 6 shows the evolution of the filament flux rope in the STEREO-B EUVI $195 \AA$ wavelength channel from 11:21:02 UT to 13:31:02 UT with solar plasma at $\approx 1.0-2.0 \mathrm{MK}$. These images also show the existence of the filament and its barbs over the western limb (see Figure 6(a)).

Cold plasma of the western part of the filament started to erupt from the middle (i.e., near the middle barb) with the brightening underneath $\approx 11: 30$ UT (see Figures 4 (b) and 5(b)).
This brightening may be due to the low-altitude reconnection in the magnetic field underneath the erupting part of the filament (see Figures 4(b), 4(c), 5(b), and 5(c)). This brightening underneath is also observed in STEREO/EUVI images, indicated by the yellow arrows (see Figures 6(a) and (b)). Thereafter, the heated filament plasma started moving in the northwest direction between $\approx 11: 20$ UT and $\approx 11: 55$ UT along the filament channel. The colder plasma in the middle of the filament was probably partly heated from episodic heating by the magnetic reconnection near its left (southeastern) foot point and partly moved from the bottom of the helical flux tubes to the top during this time (see upper panel of 

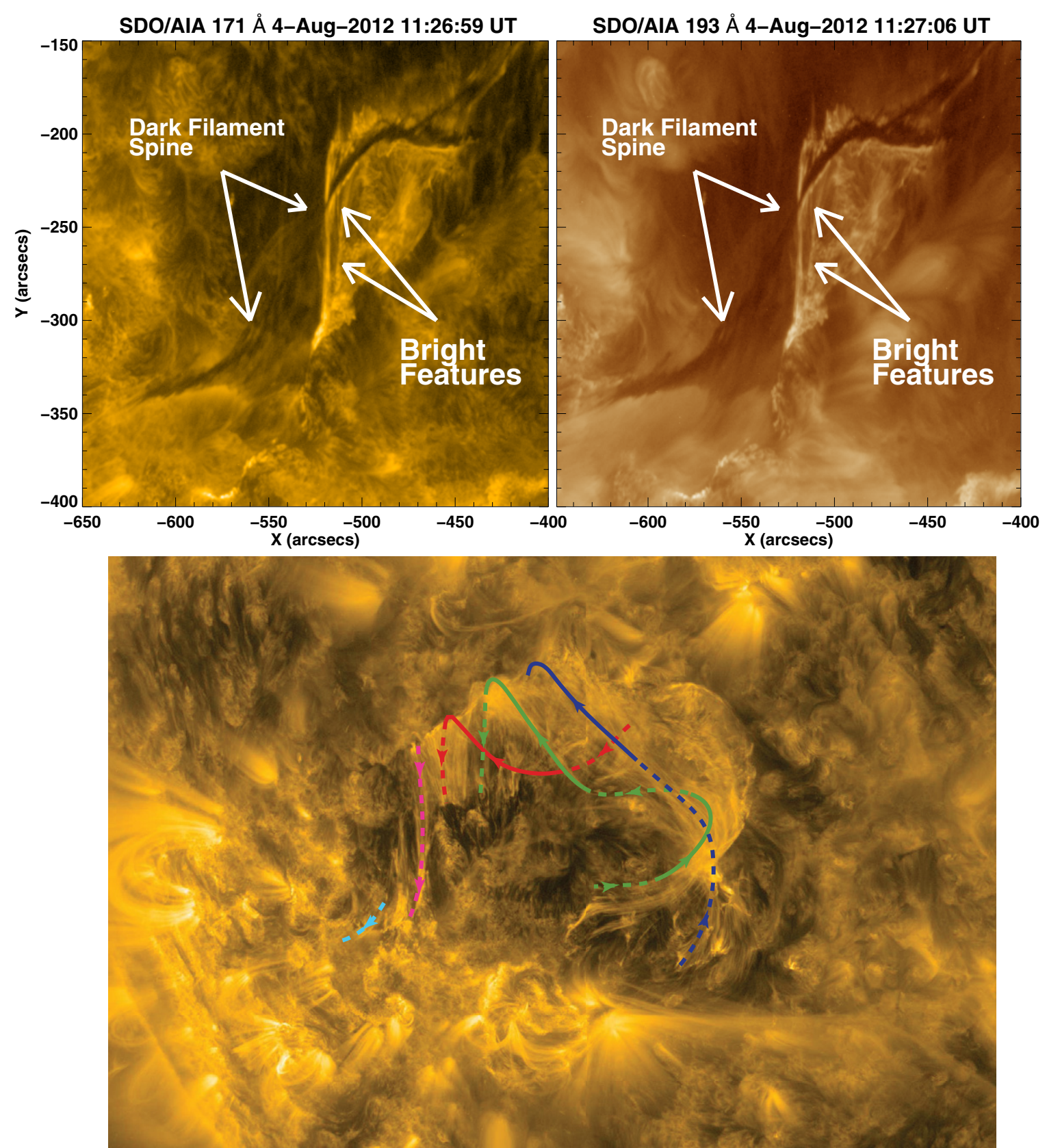

Figure 7. Upper panel: SDO/AIA 171 and $193 \AA$ images at $\approx 11: 27$ UT, showing the crossing of dark (upper) and bright (below) threads. Bottom panel: SDO/AIA $171 \AA$ Amage at 12:14:35 UT with drawn sections of flux rope field lines that can be followed from the visible threads. Field-line portions that lie above the flux rope axis are shown as solid lines, while those that lie below the flux rope axis are shown as dashed lines.

(A color version of this figure is available in the online journal.)

Figure 7). Both of these effects make this plasma invisible in the $\mathrm{H} \alpha$ line due to a decrease of absorption of radiation and the displacement of the absorption maximum from the filter band. The disappearance of the filament can be seen in the snapshot around 11:50:14 UT (see Figure 4(c)). However, it is clearly evident from the observation that the whole filament did not completely disappear. The coronal filters of SDO/AIA $171 \AA$ only provide the observation of the flux rope after the tracking of hot plasma along its various flux tubes. Some threads elongate showing the motion along the field lines, while others move laterally as a whole showing evolution of the flux rope magnetic field during the activation. Careful inspection shows that these features move below the main filament body and therefore below the flux rope axis (see upper panel of Figure 7).
Subsequently, it becomes obvious that plasma rises up on the northern side of the flux rope and then moves to the southwest above the flux rope axis. As a result, field-aligned plasma motion demonstrates a clockwise rotation when viewed along the material moving away and, therefore, a right-handed helix (see the animation related to Figure 5). This observed rotation and the sign of helicity (positive) of the flux rope containing the sinistral filament (see Figure 2) strongly support the idea of dextral/sinistral filaments being associated with flux ropes with negative/positive helicity (Chae 2000; Rust 1999). From the STEREO/EUVI limb view, the helical threads of the flux rope become clearly visible. This helically twisted flux rope has right-handed twist, represented by the tilted white arrows in Figures 6(d)-(g). From this observation, it is also clear that 

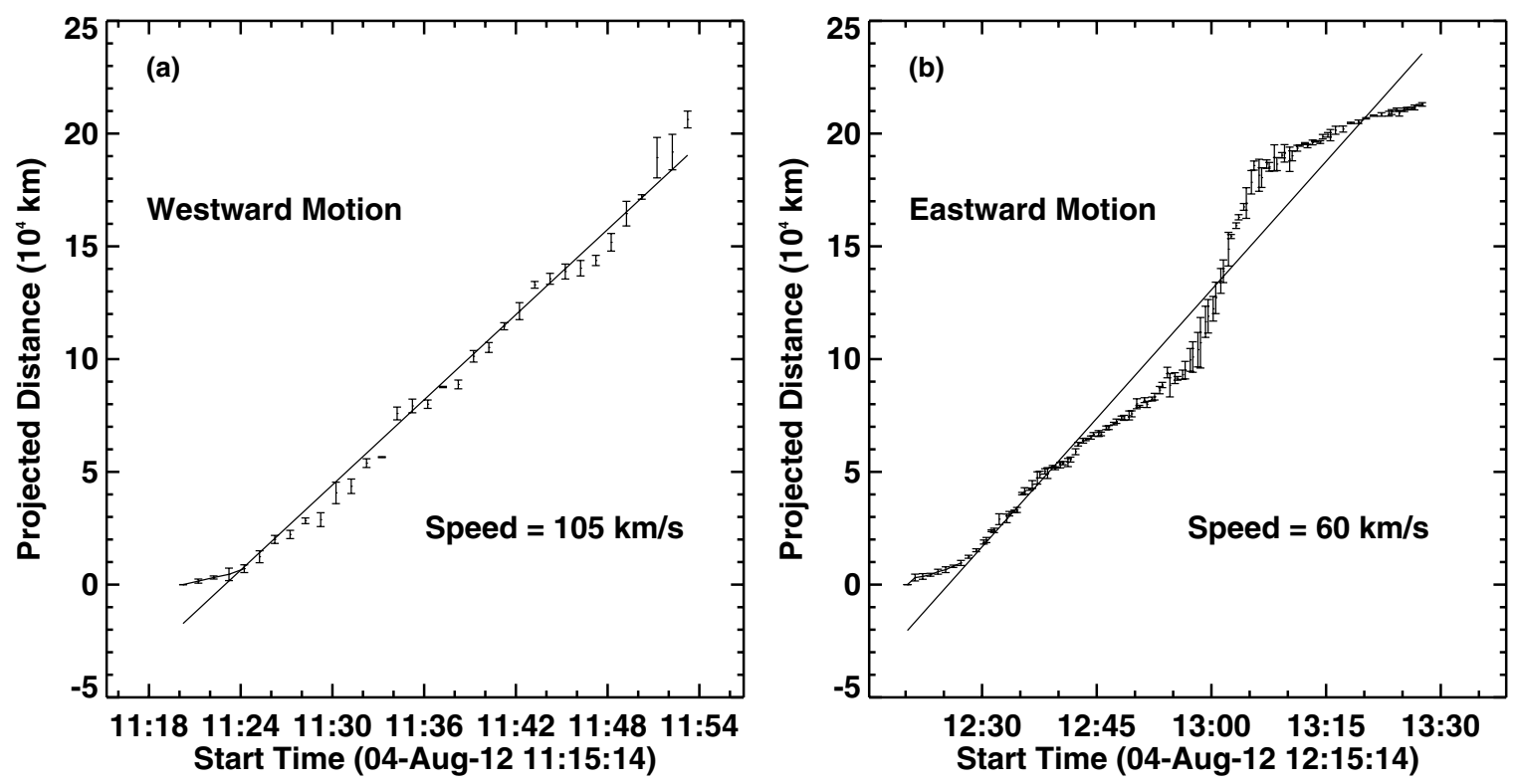

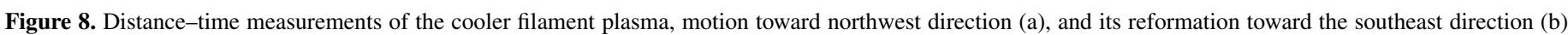

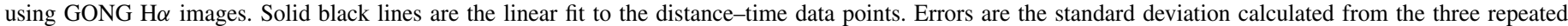

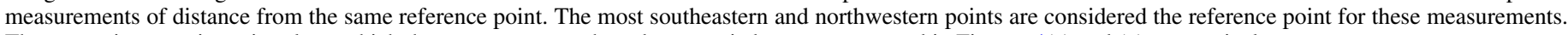
The approximate trajectories along which these measurement have been carried out are presented in Figures 4(c) and (g), respectively.

the helically twisted flux rope moves upward as the filament material moves higher (see Figures 6(d)-(g)).

The north-westward cool $\mathrm{H} \alpha$ filament motion stops at around 12:00 UT after traveling a distance of $\approx 215 \mathrm{Mm}$ (projected distance) over the solar disk (Figure 4(c)). The hot material also shows similar dynamics and stops at around 12:00 UT (Figure 5(d)). The speed of this north-westward motion is found to be $\approx 105 \mathrm{~km} \mathrm{~s}^{-1}$ (Figure 8(a)). We used GONG $\mathrm{H} \alpha$ images for these measurements. The approximate trajectory along which the distance-time measurements have been executed is plotted over the snapshot at 11:50:14 UT (see Figure 4(c)). The speed is calculated using the linear fit to these distance-time measurements in projection (Figure 8(a)). The error bars are estimated from three repeated measurements of the displacement of the same reference points. The most southeastern point is considered as the reference point for the displacement-time measurements. Then, some of the plasma moves in the southward direction (see Figures 4(e) and 5(f)), while the bulk plasma simultaneously starts returning (i.e., toward south-eastward) motion along the same channel from 12:20 UT to 13:30 UT (see Figures 4(f) and 4(g)). The whole flux rope filling with the filament material provides us an opportunity to see the whole flux rope structure. The SDO/AIA $171 \AA$ channel shows the general shape of the flux rope as a right-handed helix with the axis curved as a reverse U (see Figures 5(e) and Li \& Zhang 2013). At different times, various threads constituting the flux rope become recognizable. In the bottom panel of Figure 7, several field lines are drawn that follow visible threads of the activated flux rope. More than one turn of field lines may be expected on the whole length of the filament. The forward and backward plasma motions are observed within the magnetic flux rope. Filament plasma took $\approx 1 \mathrm{hr}$ to return from the previous location to the bottoms of field lines and restored the continuity of the filament in the $\mathrm{H} \alpha$ line with an average speed of $\approx 60 \mathrm{~km} \mathrm{~s}^{-1}$ (Figure 8(b)). The approximate trajectory along which the distance-time measurements have been executed is plotted over the snapshot at 13:10:14 UT (Figure 4(g)). This returning plasma finally regained its previous position within the filament body around $\approx 13: 30$ UT (Figure 4(h)). In addition to the dominant motions within the flux rope, the rope as a whole also showed a moderate, and later, reversing rising motion. The overall scenario of cool and hot filament plasma eruption and its reformation is clearly seen in the GONG $\mathrm{H} \alpha$ and SDO/AIA movies (see the animations associated with Figures 4 and 5). The activation of the filament, right-handed twisted structure, and filament reformation are also clearly evident in the STEREO/EUVI movie (see the animation associated with Figure 6).

Figure 9 shows the schematic scenario of the observed plasma dynamics, which we infer using GONG $\mathrm{H} \alpha, S D O / \mathrm{AIA}$ $171 \AA$ on-disk observations, and STEREO-B EUVI $195 \AA$ limb observations.

\section{CORONAL MAGNETIC FIELD CALCULATIONS AND INTERPRETATION OF THE FILAMENT FLUX ROPE STABILITY}

Despite the intensive internal motions, the flux rope (western filament) sustains stability. It does not erupt during the activation on 2012 August 4 and several days thereafter. Models of flux rope equilibrium suggest that it is stable if the surrounding magnetic field decreases with height rather slowly (van Tend \& Kuperus 1978; Molodenskii \& Filippov 1987; Filippov \& Den 2001; Kliem \& Török 2006; Démoulin \& Aulanier 2010; Guo et al. 2010). In order to investigate the magnetic field near the filament, we calculated the decay index, $n$, of the potential magnetic field, i.e.,

$$
n=-\frac{\partial \ln B_{t}}{\partial \ln h}
$$

at different heights from the photosphere (see Filippov 2013 for the details of methodology). Here, $B_{t}$ is the horizontal magnetic field component perpendicular to the flux rope axis, and $h$ is the height above the photosphere. A PIL is a place where a coronal electric current (a flux rope) can be set in horizontal equilibrium. The height of the equilibrium position depends on the strength of the electric current as well as of the coronal 


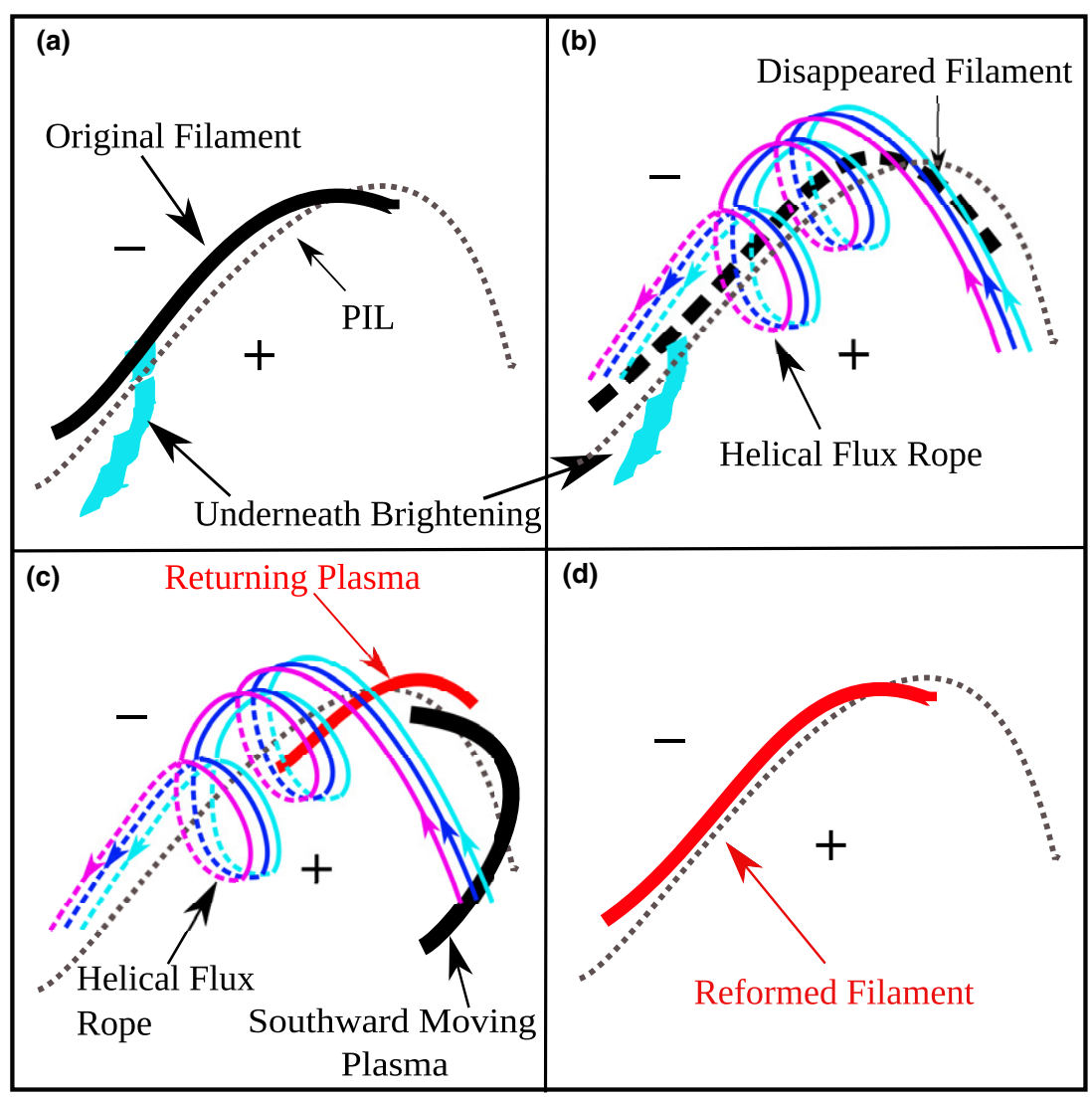

Figure 9. Schematic diagram of the event. Black/red color shows the old/reformed filaments. The dashed black line in panel (b) represents the less visible filament. The PIL is represented by the dotted black line. Helical magnetic field lines are represented by the cyan, blue, and purple colors showing the right-handed helix. Plus $(+)$ and minus (-) symbols represent the positive and negative polarity regions. Brightening underneath is represented by the sky blue patch.

(A color version of this figure is available in the online journal.)

magnetic field (Priest \& Forbes 1990; Forbes \& Isenberg 1991; Lin et al. 1998; Schmieder et al. 2013). However, for a given geometry of the flux rope axis, the stable equilibrium is possible only if the decay index of the ambient magnetic field $n$ does not exceed a critical value for a straight current channel $\left(n_{c}=1\right)$ or circular current channel $\left(n_{c}=1.5\right)$ (Filippov \& Den 2001; Kliem \& Török 2006; Démoulin \& Aulanier 2010).

In Figure 10, the thin lines show isolines corresponding to $n=0.5,1,1.5$, while thick red lines indicate the positions of PILs at three respective heights. Areas where $n>1$ are shadowed. From the STEREO/SECCHI images (Figure 6), we can measure the height above the limb of the top of the flux rope $(110 \mathrm{Mm})$ and assume that the height of the flux rope axis is about of $60 \mathrm{Mm}$ (see Figure 6). It is obvious from Figure 10 that the whole filament length at the height of $60 \mathrm{Mm}$ is within the area where $n<1$. So, the flux rope axis, even during the activation, is within the domain of stability. That may be the reason why a rather strong disturbance from the eastern eruption led only to intensive motions within the flux rope but did not cause its full eruption.

The decay index is greater at the eastern section of the PIL, as shown in Figures 10(b) and 10(c) where the eruptive eastern filament lies. The critical height is about $35 \mathrm{Mm}$, which corresponds to an average height of quiescent prominences (Dermendzhiev 1977). This provides a clue that this region might be favorable for eruption. However, we cannot substantiate it further because this section of the filament is projected on the disk as observed from SDO and STEREO spacecraft. As a result, we cannot measure the real height of the filament.

\section{RESULTS AND DISCUSSION}

In this work, we have presented the multiwavelength and multi-instrument observations of confined ejection of plasma within the filament channel, dynamical stability of the flux rope, and subsequent reformation of the erupted (disappeared) segment of the filament within the same channel. The main results of the study are as follows.

1. Our observation shows the relationship between the filament chirality (sinistral; see Figures 2 and 4(a)) and the helicity (positive) of the surrounding flux rope. The fieldaligned plasma motion also demonstrates a clockwise rotation. Thus, a right-handed helix around the flux rope axis is in agreement with the observed helicity and filament chirality.

2. The flux rope remains stable during the activation and confined eruption of the filament segment due to the location within the domain of stability (i.e., region of magnetic field decay index $n<1$ ). Hence, the properties of surrounding magnetic field play a key role in flux rope stability and the failure of the filament eruption.

3. The erupted part of the filament reforms at the initial position after confined motion along the filament channel within the flux rope. The confined eruption was possibly initiated by low-altitude reconnection underneath the eastern part of the main filament.

The filament sinistral chirality can be seen from the existence of left bearing barbs in the $\mathrm{H} \alpha$ images in Figure 2 (Martin 1998a) and deviation of thin threads within the main body of 

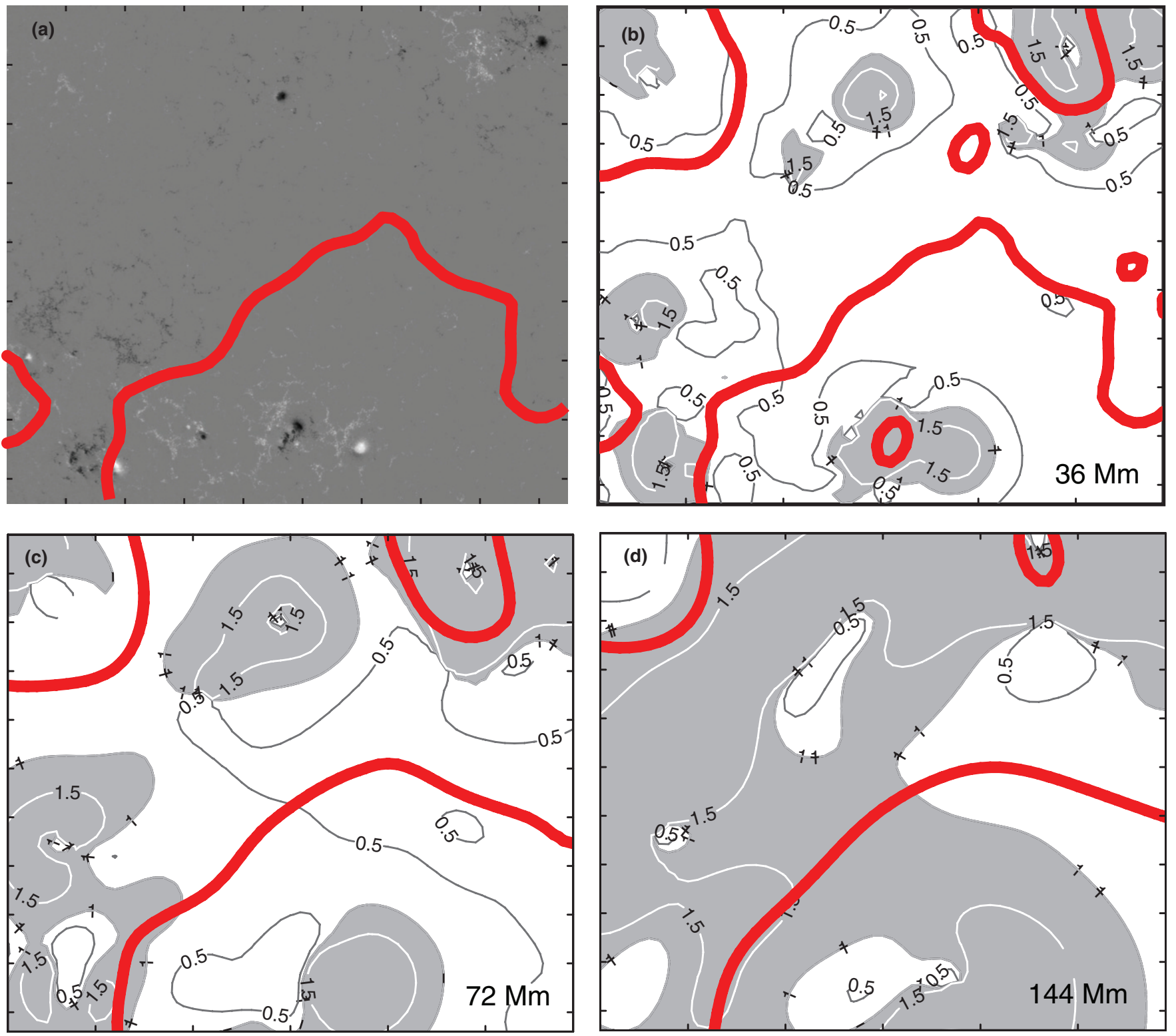

Figure 10. Fragment of the HMI magnetogram on 2012 August 4 (a) and distributions of the decay index, $n$, and PILs (thick red lines) at different heights (b, c, and d). The PIL corresponding to the filament and the flux rope at the height of $36 \mathrm{Mm}$ is superposed on the magnetogram (a). Shadowed areas show the regions where $n>1$.

(A color version of this figure is available in the online journal.)

the filament, counterclockwise from the long filament axis. We could not precisely identify the position of the true filament end points to find the magnetic polarity of each of them. However, from the EUV images after the filament activation (Figure 5), we conclude that the right end of the filament channel is anchored in an area of predominantly positive polarity, and individual flux rope threads are anchored by their western ends with magnetic features of positive polarity (see Figure 4 of $\mathrm{Li} \&$ Zhang 2013), in accordance with the sinistral chirality of the channel. Our filament lies in the southern hemisphere and shows sinistral chirality, which is in agreement with the well-known hemispheric chirality rule (Pevtsov et al. 2003).

From the general structure of the activated flux rope and motions within, we determine the positive sign of its helicity. Chae (2000) has given yet another method to infer the helicity of a filament from the crossing geometry of bright and dark threads in the filament in EUV wavelength. In our case, from the
SDO/AIA observations (see Figure 7, upper panel), we find such crossing of underlying bright and overlying dark threads during the activation of the filament. This structure is similar to the geometry of crossing type I mentioned by Chae (2000), which corresponds to the positive helicity. The $S D O /$ AIA observations of the field-aligned plasma motions show a clockwise rotation of plasma moving away from an observer, which corresponds to a right-handed helix. Thus, our observation is an example of the filament chirality (sinistral) and helicity (positive) of the surrounding flux rope.

Partial and failed eruptions have been studied in the past to find the possible physical interpretation for these kinds of eruptions (Ji et al. 2003; Török \& Kliem 2005; Liu et al. 2009; Kumar et al. 2011; Joshi et al. 2013a). In some of the studies, the overlying magnetic field is considered as the cause of the failed eruption (Ji et al. 2003; Kumar et al. 2011; Mrozek 2011), despite the evolution of high twists and different kinds of 
instabilities (Kumar et al. 2010; Srivastava et al. 2010, 2013, and references cited therein). Liu et al. (2009) interpreted the failed filament eruption as the result of an asymmetric confinement of the ambient field above the filament channel. Török \& Kliem (2005) simulated the failed eruption and interpreted it in terms of a slowly decreasing gradient of the overlying magnetic field with height. Recently, Filippov (2013) investigated a filament eruption and found that it started only after reaching a height in the corona corresponding to a decay index greater than one. We also calculated the potential magnetic field decay index $n$ at different heights near the filament. We found that the whole filament, at a height of about $60 \mathrm{Mm}$, is within the domain of stability (i.e., $n<1$ ) even during its activation phase. This is the possible cause for the stability of the filament and its reformation. After heating and displacement from the bottoms of the helical field tubes to their tops, filament plasma cools again and returns to the previous location, reforming the filament continuity. Recently, Li \& Zhang (2013) discussed the fine structure of the flux rope on 2012 August 4 associated with the same filament and found that each end the flux rope was composed of multiple foot points anchored in opposite polarities. However, they did not aim to study the filament stability and its reformation, which we have investigated in the present paper.

In addition, it is also interesting to investigate the cause of activation of the eastern part of a stable filament. We suggest that the CME following the eruption of the eastern section perturbs the overlying large-scale coronal field, and then the equilibrium of the western section may be perturbed as well. As a result, the western section begins to move to a new equilibrium at a different height. With the recovery of the large-scale coronal field, the original equilibrium of the western section may be re-established close to the original height. This situation is different from the one described by Guo et al. (2010), who found a filament located in an unstable height range (explaining the onset of the eruption) with a height range of stability lying above (explaining the failure of the eruption).

Koleva et al. (2012) observed the partial reformation within two days after the prominence eruption on 2010 March 31. Tripathi et al. (2009) also discussed the reformation of the cavity (within 20 minutes to 1 day) after the partial eruption of filaments. In our case, the reformation of filament within the same channel shows that the magnetic configuration supporting the filament is not destroyed during the filament activation. The filament was reformed within $\approx 2 \mathrm{hr}$. This rapid reformation is also a unique observation that has not been previously reported. The location of the filament flux rope within the region of stability with coronal magnetic field decay index $n<1$ is most likely the cause of this fast reformation of the filament.

If this huge filament lying near the center of the disk were fully eruptive, it would have served as the cause of severe outer coronal responses (e.g., halo CME, solar energetic particles, and space weather effects). However, it did have sufficient reserve of stability to preserve its full eruption, despite observed intensive internal plasma dynamics due to the ambient coronal magnetic field. Therefore, such studies of filament stabilization conditions are very important to forecast the initial conditions for their eruptions and thereby to forecast associated space weather conditions.

The authors thank the referee for constructive comments and suggestions. The authors thank the Indo-Russian (DST-RFBR) Project on the Study of Dynamical Events in the Solar Atmosphere during the Maximum of Solar Cycle 24 (INT/RFBR/P-117, RFBR 12-02-00008, and 14-02-92690) for its support to this study during our bilateral collaboration. We thank the SDO/AIA, STEREO/SECCHI, GONG, and BBSO teams for providing their data for the present study. N.C.J. thanks Aryabhatta Research Institute of Observational Sciences (ARIES), Nainital for providing a postdoctoral grant. We also acknowledge the partial support of the IUSSTF/JC-Solar Eruptive Phenomena/99-2010/2011-2012 project. R.C., A.K.S., and W.U. acknowledge ISRO/RESPOND Project no. ISRO/ RES/2/379/12-13. A.K.S. acknowledges Prof. S.F. Martin for significant discussions of the scientific aspects of this event, and S. Srivastava for encouragement during this study.

\section{REFERENCES}

Brueckner, G. E., Howard, R. A., Koomen, M. J., et al. 1995, SoPh, 162, 357 Chae, J. 2000, ApJL, 540, L115

Chandra, R., Pariat, E., Schmieder, B., Mandrini, C. H., \& Uddin, W. 2010, SoPh, 261, 127

Chandra, R., Schmieder, B., Aulanier, G., \& Malherbe, J. M. 2009, SoPh, 258, 53

Chandra, R., Schmieder, B., Mandrini, C. H., et al. 2011, SoPh, 269, 83

Chen, P. F. 2011, LRSP, 8, 1

Choudhary, D. P., Gosain, S., Gopalswamy, N., et al. 2013, AdSpR, 52, 1561

Choudhary, D. P., \& Moore, R. L. 2003, GeoRL, 30, 2107

Démoulin, P., \& Aulanier, G. 2010, ApJ, 718, 1388

Dermendzhiev, V. 1977, AISof, 2, 8

DeVore, C. R., \& Antiochos, S. K. 2008, ApJ, 680, 740

Fan, Y. 2010, ApJ, 719, 728

Filippov, B. 2013, ApJ, 773, 10

Filippov, B., \& Koutchmy, S. 2002, SoPh, 208, 283

Filippov, B. P., \& Den, O. G. 2001, JGR, 106, 25177

Filippov, B. P., Gopalswamy, N., \& Lozhechkin, A. V. 2001, SoPh, 203, 119

Forbes, T. G., \& Isenberg, P. A. 1991, ApJ, 373, 294

Gaizauskas, V., Mackay, D. H., \& Harvey, K. L. 2001, ApJ, 558, 888

Gaizauskas, V., Zirker, J. B., Sweetland, C., \& Kovacs, A. 1997, ApJ, 479, 448

Gilbert, H. R., Alexander, D., \& Liu, R. 2007, SoPh, 245, 287

Guo, Y., Ding, M. D., Schmieder, B., et al. 2010, ApJL, 725, L38

Harvey, J. W., Bolding, J., Clark, R., et al. 2011, in Bulletin of the American Astronomical Society, Solar Physics Division, Meeting, 42, 1745

Howard, R. A., Moses, J. D., Vourlidas, A., et al. 2008, SSRv, 136, 67

Ji, H., Wang, H., Schmahl, E. J., Moon, Y.-J., \& Jiang, Y. 2003, ApJL, 595, L135

Joshi, N. C., Srivastava, A. K., Filippov, B., et al. 2013a, ApJ, 771, 65

Joshi, N. C., Uddin, W., Srivastava, A. K., et al. 2013b, AdSpR, 52, 1

Kliem, B., \& Török, T. 2006, PhRvL, 96, 255002

Koleva, K., Madjarska, M. S., Duchlev, P., et al. 2012, A\&A, 540, A127

Kumar, P., Srivastava, A. K., Filippov, B., Erdélyi, R., \& Uddin, W. 2011, SoPh, 272, 301

Kumar, P., Srivastava, A. K., Filippov, B., \& Uddin, W. 2010, SoPh, 266, 39

Kuridze, D., Mathioudakis, M., Kowalski, A. F., et al. 2013, A\&A, 552, A55

Labrosse, N., Heinzel, P., Vial, J.-C., et al. 2010, SSRv, 151, 243

Lemen, J. R., Title, A. M., Akin, D. J., et al. 2012, SoPh, 275, 17

Li, T., \& Zhang, J. 2013, ApJL, 770, L25

Lin, J., Forbes, T. G., Isenberg, P. A., \& Demoulin, P. 1998, ApJ, 504, 1006

Lites, B. W. 2005, ApJ, 622, 1275

Lites, B. W., \& Low, B. C. 1997, SoPh, 174, 91

Liu, Y., Su, J., Xu, Z., et al. 2009, ApJL, 696, L70

Mackay, D. H., Gaizauskas, V., \& van Ballegooijen, A. A. 2000, ApJ, 544, 1122

Mackay, D. H., Karpen, J. T., Ballester, J. L., Schmieder, B., \& Aulanier, G. 2010, SSRv, 151, 333

Martin, S. F. 1998a, SoPh, 182, 107

Martin, S. F. 1998b, in ASP Conf. Ser. 150, IAU Colloq. 167: New Perspectives on Solar Prominences, ed. D. F. Webb, B. Schmieder, \& D. M. Rust (San Francisco, CA: ASP), 419

Molodenskii, M. M., \& Filippov, B. P. 1987, SvA, 31, 564

Mrozek, T. 2011, SoPh, 270, 191

Muglach, K., Wang, Y.-M., \& Kliem, B. 2009, ApJ, 703, 976

Pevtsov, A. A., Balasubramaniam, K. S., \& Rogers, J. W. 2003, ApJ, 595, 500

Priest, E. R., \& Forbes, T. G. 1990, SoPh, 126, 319

Rust, D. M. 1999, GMS, 111, 221

Rust, D. M. 2001, JGR, 106, 25075

Schmieder, B., Chandra, R., Berlicki, A., \& Mein, P. 2010, A\&A, 514, A68 
Schmieder, B., Démoulin, P., \& Aulanier, G. 2013, AdSpR, 51, 1967

Schmieder, B., Malherbe, J. M., \& Raadu, M. A. 1985, A\&A, 142, 249

Schou, J., Scherrer, P. H., Bush, R. I., et al. 2012, SoPh, 275, 229

Shen, Y., Liu, Y., \& Su, J. 2012, ApJ, 750, 12

Srivastava, A. K., Erdélyi, R., Tripathi, D., et al. 2013, ApJL, 765, L42

Srivastava, A. K., Zaqarashvili, T. V., Kumar, P., \& Khodachenko, M. L. 2010, ApJ, 715, 292
Sterling, A. C., Harra, L. K., \& Moore, R. L. 2007, ApJ, 669, 1359

Sterling, A. C., Moore, R. L., \& Hara, H. 2012, ApJ, 761, 69

Török, T., \& Kliem, B. 2005, ApJL, 630, L97

Tripathi, D., Gibson, S. E., Qiu, J., et al. 2009, A\&A, 498, 295

van Tend, W., \& Kuperus, M. 1978, SoPh, 59, 115

Wuelser, J.-P., Lemen, J. R., Tarbell, T. D., et al. 2004, Proc. SPIE, 5171, 111 